Article

\title{
Acute Effects of Polyphenols from Cranberries and Grape Seeds on Endothelial Function and Performance in Elite Athletes
}

\author{
Kim Labonté, Charles Couillard, Annie Motard-Bélanger, Marie-Eve Paradis, Patrick Couture and \\ Benoît Lamarche*
}

The Institute on Nutrition and Functional Foods (INAF), Laval University, Québec G1V 0A6, Canada; E-Mails: kim.labonte@videotron.ca (K.L.); Charles.couillard@ fsaa.ulaval.ca (C.C.); Annie.MotardBelanger@dfc-plc.ca (A.M.-B.); marie-eve.paradis@fsaa.ulaval.ca (M.-E.P.); patrick.couture@crchul.ulaval.ca (P.C.)

* Author to whom correspondence should be addressed; E-Mail: benoit.lamarche@inaf.ulaval.ca; Tel.: +1-418-656-2131 extension 4355; Fax: +1-418-656-5877.

Received: 11 June 2013; in revised form: 3 July 2013 / Accepted: 3 July 2013 /

Published: 12 July 2013

\begin{abstract}
We examined how intake of polyphenols modifies brachial artery flow-mediated dilation (FMD) at rest, and cycling anaerobic performance, in elite athletes. In the first randomized cross-over study, FMD was measured over a three-hour period on two occasions in eight elite male and female athletes after acute consumption of either polyphenols from cranberries and grape seeds (600 mg) or a polyphenol-free placebo drink. Consumption of the polyphenol-rich drink led to a significant increase in FMD compared to placebo $(p=0.02)$, with a peak at $60 \mathrm{~min}$. In a second study, 12 elite male and female athletes completed a threekilometer time trial (TT) on an ergocycle on two occasions in random order, either after consumption of $800 \mathrm{mg}$ of polyphenols or a placebo. Acute intake of the polyphenol extract had no impact on the three-kilometer time trial completion. However, plasma lactate levels were significantly lower before and after the TT when subjects consumed the polyphenols vs. placebo $(p<0.05)$. Results suggest that polyphenols from cranberries and grape seeds acutely modifies FMD at rest in elite athletes but this does not translate into enhanced cycling anaerobic performance.
\end{abstract}


Keywords: polyphenol; athlete; endothelial function; FMD

\section{Introduction}

Berry fruits contain a large variety of polyphenolic compounds that have been characterized as having numerous physiological effects in humans. Intake of dietary polyphenols not only enhances whole body antioxidant capacity [1], but also modulates endothelial function [2]. Polyphenols from various sources have also been shown to have anti-inflammatory properties in non-athletes and in patients with coronary heart disease [3].

Increased oxygen consumption through physical activity and exercise training, particularly at high intensities, leads to a transient pro-oxidative and pro-inflammatory state [4]. There is also accumulating evidence suggesting that oxidative stress may hamper sports performance, induce fatigue, and delay recovery [5-7]. This has led many athletes to use antioxidant supplements, despite the fact that there is currently no convincing evidence supporting the beneficial effects of antioxidant supplementation on performance and exercise recovery [8].

On the other hand, it is not clear how dietary intake of polyphenols affects endothelial function in athletes, as most studies that have investigated this topic have been conducted in non-athletes as well as patients with chronic disease. Furthermore, the potential of polyphenols to improve blood flow must not be overlooked as restricted blood flow is considered a key limiting factor in muscle oxygenation during high intensity efforts [9]. For instance, it has been shown that restricted blood flow is an important determinant of performance in speed skaters [10]. However, information remains scarce regarding the impact of nutritional products on performance via their impact on vascular function, endothelial function and blood flow [11]. It is also not clear how dietary intake of polyphenols affects endothelial function in athletes, as most studies that have investigated this topic have been conducted in non-athletes as well as patients with chronic disease.

The objective of this study was to test the impact of a polyphenol extract from grape seed and cranberries on endothelial function and performance in elite athletes. We hypothesized that consumption of a high dose of polyphenol from cranberry and grape seeds improves vascular function in highly trained athletes and that this, in turn, improves performance on a maximal three-kilometer cycling time-trial.

\section{Subjects and Methods}

This investigation comprised two studies each undertaken according to a randomized, double blind, placebo-controlled, cross-over design. In the first study, flow-mediated dilation (FMD) at rest was measured over a three-hour period on two occasions in elite athletes, after acute consumption of a sports drink added with a proprietary blend of polyphenols from cranberries and grape seeds vs. a carbohydratematched polyphenol-free placebo drink. In the second study, the performance of athletes on a three- 
kilometer time trial (TT) was investigated on two occasions, i.e., after consumption of the polyphenol-rich drink $v s$. after consumption of a placebo.

\subsection{Population}

Elite athletes living in the Quebec City Metropolitan area were recruited to participate in this study. Participants were members of National or Development Teams in Canada, or were defined as elite based on criteria from sport specific Quebec Federations. In study \#1, 6/8 athletes were speed skaters, one was a biathlete, and one was a cyclist. In study \#2, nine were speed skaters; the others were cyclists (2) or crosscountry skiers (1). All athletes trained more than $20 \mathrm{~h}$ a week, were free of cardiovascular diseases, diabetes or endocrine disorders, hypercholesterolemia, iron-deficiency, or megaloblastic anemia (according to their physicians), did not consume alcohol in excess (more than two drinks by day for men, one for women), or had allergies to cranberry. Data on $\mathrm{VO}_{2} \mathrm{max}$ were neither collected nor available. Subjects gave their written consent to participate in this study, which was approved by the Laval University Ethics Committee.

\subsection{Nutritional Habits}

Before both studies, subjects completed a validated food frequency questionnaire [12] with a registered dietician to assess usual food intake and to provide nutritional guidance specific to the purposes of the study. Subjects were asked to avoid cranberry juice and derived products during the entire experimental periods, while also limiting their intake of polyphenols-rich foods (e.g., berries, onions, broccoli, apples, red grapes, and fruit juices). The use of polyphenol supplements was strictly forbidden during both studies. Coffee and tea were not permitted on tests days. Participants also had to refrain from drinking alcoholic beverages $72 \mathrm{~h}$ prior to each test.

\subsection{Study Designs}

\subsubsection{Study 1: Polyphenols and FMD in Elite Athletes}

Acute changes in FMD were measured after administration of the following treatments in random order, (1) $600 \mathrm{mg}$ of a proprietary blend of polyphenols from cranberries (Vaccinium macrocarpon) and grape seeds (Vitis vinifera) commercialized as CranLoad ${ }^{\mathrm{TM}}$, mixed in $500 \mathrm{~mL}$ of water, and (2) after polyphenol-free carbohydrate-matched and flavor-matched placebo drink $(500 \mathrm{~mL})$. Both beverages contained equal quantities of carbohydrates $(6 \%)$ from identical sources. FMD was assessed over a threehour period at pre-determined time points $(0,30,60,90,120,150$, and $180 \mathrm{~min})$.

Flow mediated vasodilation (FMD) was assessed by ultrasonography, as previously described [13]. Briefly, the brachial artery was scanned in the longitudinal plane, above the antecubital fossa with a highresolution, linear array ultrasonic transducer of 7.5-10.5 MHz (Hewlett-Packard, Sonos 5500, Andover, Massachusetts, USA). Reactive hyperaemia was induced by inflating a forearm occlusive cuff to $220 \mathrm{~mm}$ 
$\mathrm{Hg}$ for $3 \mathrm{~min}$. Brachial artery diameter at each time point was measured $60 \mathrm{~s}$ and $90 \mathrm{~s}$ after cuff deflation. Because both measurements (60 and $90 \mathrm{~s}$ ) yielded very similar results, the mean of the two assessments was used in the present analysis. All scans were performed by the same examiner throughout the study. Images were digitally acquired and analyzed offline by two independent observers, who were blinded to the subject's treatment assignment. Data from each person were comparable and therefore the mean of the two independent assessments was used for the analysis. FMD was calculated as the percentage increase in hyperaemia-induced brachial artery diameter from values measured at rest. The area under the curve of the FMD response over time was calculated using the trapezoid method [14]. The AUC provides an integrated measure of the change in the outcome measure over time, and has been used in the past to characterize the FMD response to various treatments and challenges [14,15]. Both tests (placebo and polyphenol-rich drink) were undertaken one week apart in each athlete but on the same day of their weekly training program. FMD tests were performed in the morning, in a fasting state. One subject had to be excluded from the analysis because of the quality of the images collected, which did not allow the measurement of arterial diameter with reliability. Analysis of FMD in response to the test drinks was therefore performed on a total of eight subjects.

Plasma samples were taken over the three-hour FMD test at pre-determined time points $(0,15,30,45$, $60,90,120,150$, and $180 \mathrm{~min}$ ) for the assessment of plasma glucose, insulin, and C-peptide, using a catheter inserted in the arm opposite to the one used for FMD testing.

\subsubsection{Study 2: Polyphenols and Performance}

Participants completed 3-km cycling time trials (TT) in order to investigate the impact of polyphenol intake on acute peak performance. For that purpose, participants came to the Physical Activity Sciences Laboratory of Laval University on three separate occasions, each time after lunch. The first test was undertaken in order for athletes to get familiar with the experimental procedures and the nature of the test. The 3-km TT was repeated twice thereafter by all participants in random order, i.e., once after the consumption of $800 \mathrm{mg}$ of the polyphenol extract taken with $60 \mathrm{~mL}$ of water, and once after the consumption of $60 \mathrm{~mL}$ of a flavor-matched placebo drink that contained no polyphenols. Seven subjects performed the TT using the placebo first and five did the TT using the polyphenol supplement first. The dose of polyphenols in the performance test was increased (800 vs. $600 \mathrm{mg}$ in Study \#1) to maximize the impact on FMD, thereby increasing the probability to obtain a significant result on performance. No carbohydrate was added to these experimental drinks. Because the 3-km TT was performed after lunch, nutritional directions were given to subjects to ensure that they consumed the same breakfast, lunch, and snack before each test was comparable. Each test was separated by one week and participants as well as all the laboratory staff were blinded from treatment assignment.

The 3-km TTs were performed on a Velotron cyclePro (RacerMate Inc., Seattle, WA, USA). The polyphenol-rich drink and the placebo were taken $60 \mathrm{~min}$ prior to the 3-km TT. Subjects had a $30 \mathrm{~min}$ period for warm up, during which they were only allowed to drink water. Athletes were allowed to select their own pace and wheel resistance, which they could change freely during the test and also had the 
possibility to pedal in a standing rather than sitting position. The $3-\mathrm{km}$ test was run on a simulated flat course. Time to completion was the primary outcome. Other measures included generated wattage, heart rate, plasma lactate levels, and perceived exertion. Plasma lactate concentrations were measured after the warm up as well as right at the end of the $3-\mathrm{km}$ TT and 2.5 and 5 min post TT with a finger stick device and a lactate meter (Lactate Pro LT-1710, Fact-Canada, Quesnel, BC, Canada). Perceived exertion was assessed, both for globally perceived effort, as well as specifically for legs and breathing using the 20point Borg scale. During each test, coaches and training partners were allowed to support the participants. Heart rate was monitored throughout the test with an electrode strap belt. Prevalence of side-effect was assessed by a questionnaire completed on the day that followed each 3-km TT test.

\subsection{Statistical Analyses}

Data are expressed as mean \pm standard deviation unless specified otherwise. Between treatments comparisons in both studies were tested using mixed models for repeated measurements (SAS software version 9.1). In study \#1, the primary analysis was to compare the area under the curve (AUC) of the change over time in the dependent variables (FDM- primary outcome, insulin and glucose- secondary outcomes) between the two treatments (polyphenols vs. placebo). The model included treatment as fixed and repeated measure effect and subjects as random effect. The covariance matrix structure providing the best fit to the model based on the Akaike Information Criterion was retained. If a significant treatment effect was noted, pairwise comparison of values on each treatment was undertaken for each time point as post hoc analyses, with no adjustment for multiple comparisons given the small sample size. Interaction of treatment by time was also not considered due to limited statistical power in this relatively small sample size. We also did not consider any other potential interaction (e.g., with sex) for the same reason. Analyses in study \#2 were performed similarly, with treatment (polyphenols vs. placebo) as fixed effect (repeated measure) and subject as random effect. Primary endpoint was the time required to complete the $3 \mathrm{~km}$ TT. For other secondary outcomes (e.g., lactate levels), each time was analyzed separately. A P value less than 0.05 was considered statistically significant. 


\section{Results}

Characteristics of the participants are presented in Table 1.

Table 1. Subjects' characteristics at screening.

\begin{tabular}{lcc}
\hline Variable & Study $\mathbf{1}$ & Study 2 \\
\hline Number of subjects & 8 & 12 \\
Females $(\mathrm{N})$ & 4 & 2 \\
Age $($ years) & $23.4 \pm 7.7$ & $22.0 \pm 2.4$ \\
Weight $(\mathrm{kg})$ & $70.8 \pm 6.7$ & $75.7 \pm 10.4$ \\
Height $(\mathrm{m})$ & $1.75 \pm 0.08$ & $1.79 \pm 0.07$ \\
BMI $\left(\mathrm{kg} / \mathrm{m}^{2}\right)$ & $23.2 \pm 0.8$ & $23.6 \pm 1.9$ \\
Energy $(\mathrm{kcal})$ & $3931 \pm 1347$ & $3139 \pm 735$ \\
\% from carbohydrates & $57 \pm 7$ & $55 \pm 6$ \\
\% from protein & $16 \pm 2$ & $17 \pm 3$ \\
\% from fat & $26 \pm 5$ & $28 \pm 6$ \\
\hline
\end{tabular}

Values are presented as mean \pm standard deviation. BMI: body mass index; $33 \%$ (4/12) of subjects in study \#2 have also participated in study \#1.

Figure 1. Flow-mediated dilation (FMD) variation from baseline after intake of the polyphenol-rich drink (grey symbols) and placebo (black symbols) in elite athletes. $* p<0.05$ versus baseline and placebo at specified time points. The insert presents the area under the curve (AUC) of the change in FMD over time above baseline (time 0 ) values. The $\mathrm{Y}$ axis is in $\% * \min$.

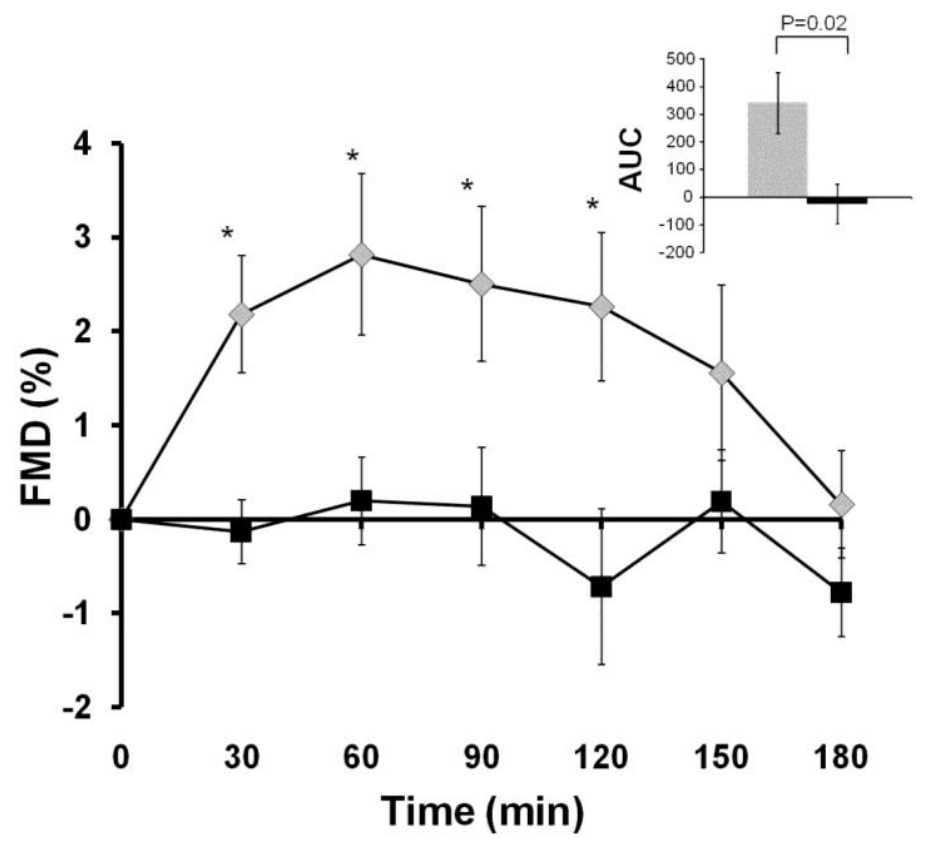




\subsection{Study 1- Polyphenols and FMD}

The AUC of FMD over time was greater after the polyphenol-rich drink than after the placebo $(p=0.02)$ (Figure 1). Intake of the polyphenol-rich drink significantly increased FMD at times 30, 60, 90, and 120 min following hyperaemia compared with placebo $(p<0.05)$. FMD peaked in average at 60 min. Mean FMD returned to baseline values three hours after ingestion of the polyphenol rich drink. As shown in Figure 2, there was no difference in plasma glucose, insulin, and C-peptide responses to the polyphenol-rich and placebo drinks.

Figure 2. Glycemia, insulinemia, and C-Peptide concentration at baseline and after the ingestion of the polyphenol-rich drink or placebo in elite athletes. The inserts present the area under the curve (AUC) of the change over time above baseline (time 0 ) values. The Y axis for the inserts is in $\mathrm{mmol} / \mathrm{L}^{*} \mathrm{~min}$. There was no statistical difference between treatments.
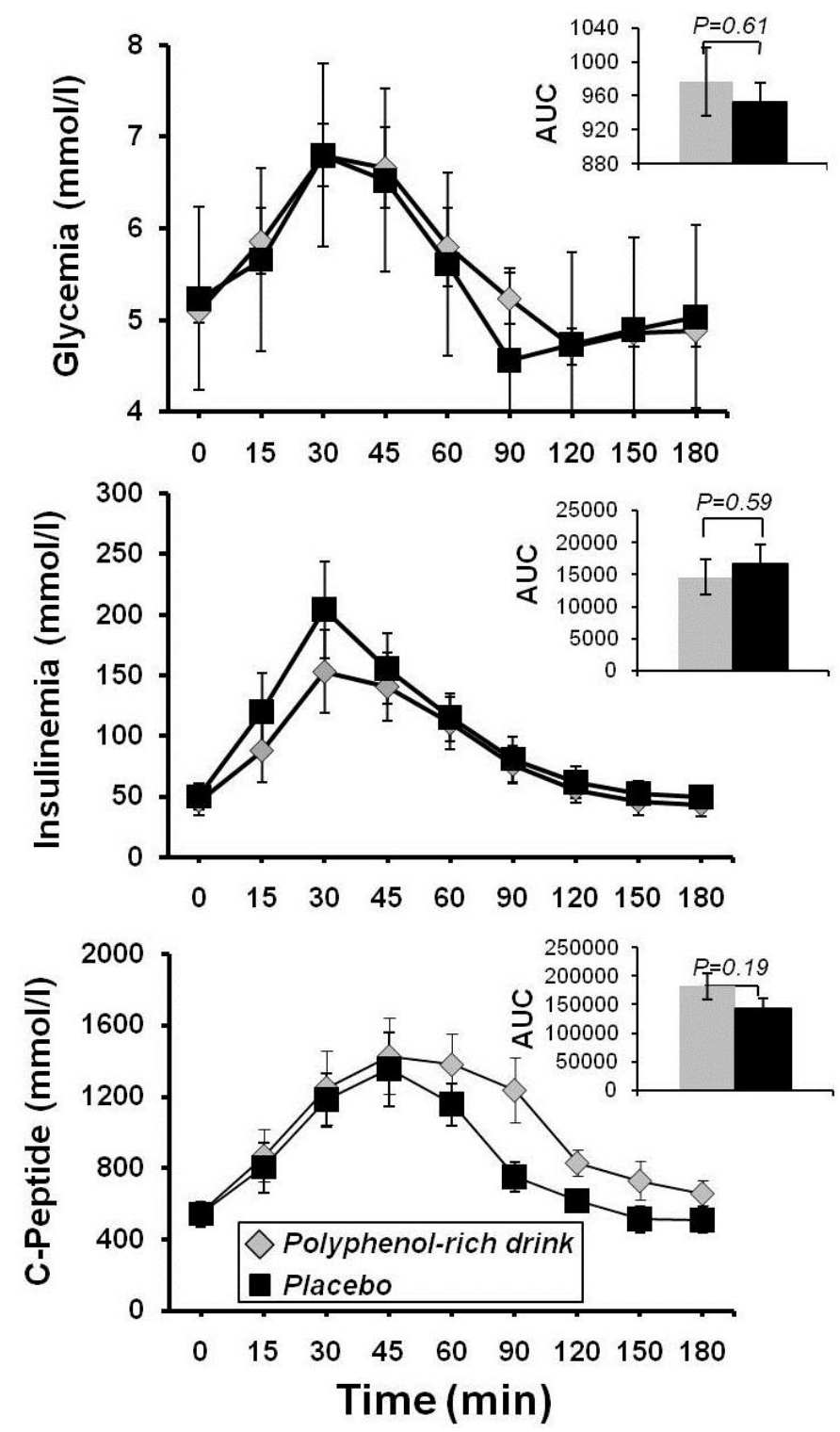


\subsection{Study 2-Polyphenols and Performance}

Time to completion of the 3-km TT was virtually identical between the two treatments $(267.5 \pm 24.5$ s vs. $265.7 \pm 21.4 \mathrm{~s}$ with the polyphenol-rich and placebo treatments respectively) and the difference between the two $(1.75 \mathrm{~s}, 0.66 \%)$ was not significant $(p=0.27)$. There was also no difference in average wattage and perceived exertion after the TT between the polyphenol-rich and placebo drinks. There was no apparent indication that sequence of treatments (placebo first or polyphenol first) had any influence on the outcomes (not shown). However, consumption of polyphenols 60 min before the TT was associated with lower plasma lactate concentrations both before the onset of the test $(-0.7 \mathrm{mmol} / \mathrm{L}$, $-30.3 \%)$, as well as immediately following the test $(-1.0 \mathrm{mmol} / \mathrm{L},-7.9 \%)$, and $2.5 \mathrm{~min}$ post recovery $(-0.7 \mathrm{mmol} / \mathrm{L},-4.8 \%$, Figure $3, p<0.05)$. Heart rates $(\mathrm{HR})$ at the end of the $3-\mathrm{km}$ TT were comparable after both conditions (polyphenol-rich drink: $188 \pm 6$ bpm, placebo: $190 \pm 6$ bpm, $p=0.22$ ). However, HR was significantly lower $2 \min$ post exercise $(140 \pm 10 \mathrm{vs} .144 \pm 9 \mathrm{bpm}, p=0.04, \mathrm{~N}=11)$ and 5 min post exercise (120 \pm 7 vs. $122 \pm 8 \mathrm{bpm}, p=0.05, \mathrm{~N}=10)$ after the polyphenol-rich drink $v s$. placebo. No side effect was reported by the athletes in response to either treatment.

Figure 3. Blood lactate levels before and immediately after the $3-\mathrm{km}$ time trial (TT) test and $2.5 \mathrm{~min}$ and $5 \mathrm{~min}$ post effort.

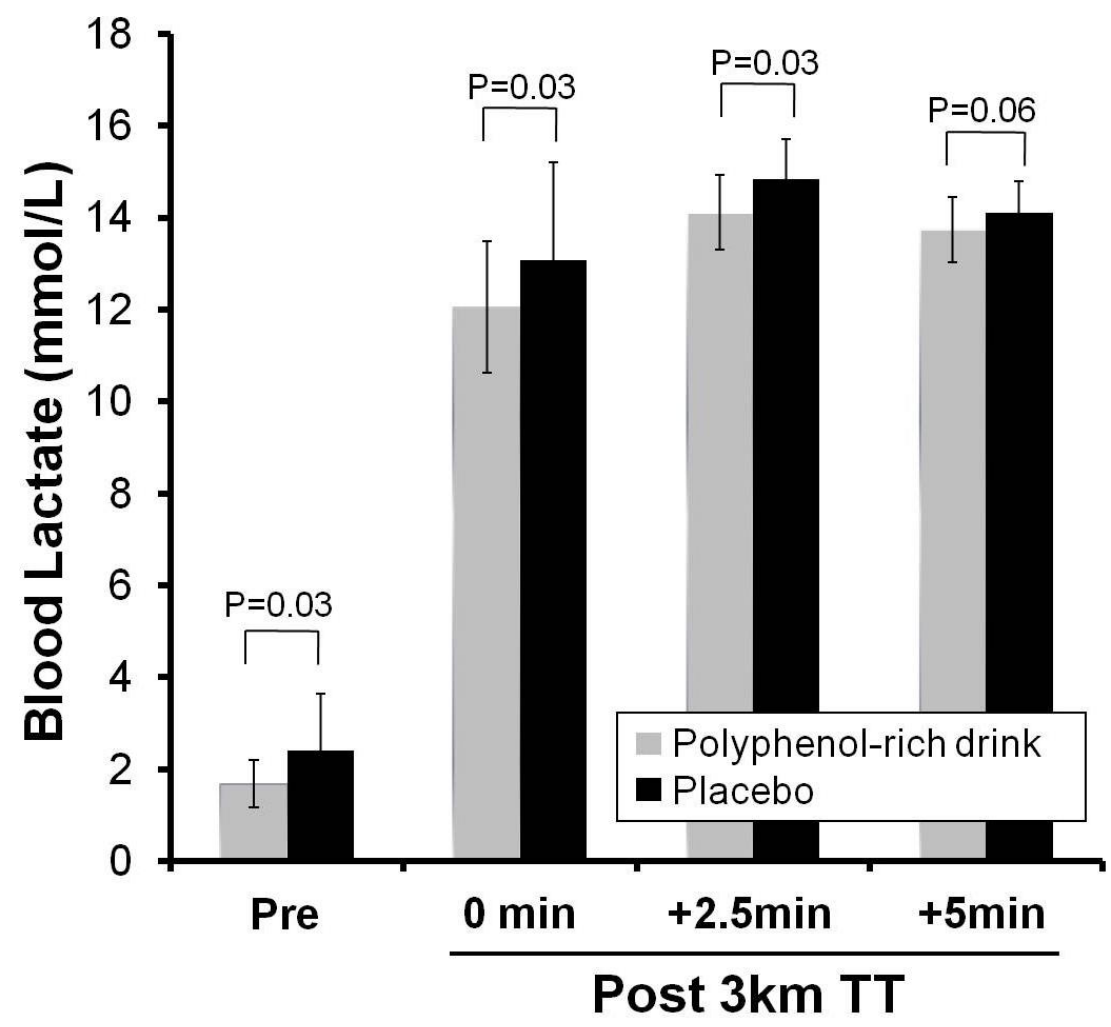




\section{Discussion}

The present study suggests that consumption of a polyphenol-rich drink significantly increases FMD in elite athletes. Although this change in vascular function is not associated with enhanced cycling performance, blood lactate and heart rate post TT were lower with polyphenols than with placebo.

\subsection{Study 1- Polyphenols and FMD}

The significant increase in acute FMD following consumption of the polyphenol-rich drink is consistent with other studies conducted in non-athlete populations [16-19]. For instance, FMD was increased after consumption of $477 \mathrm{mg}$ of polyphenols from non-alcoholized red wine in healthy subjects [18]. Coronary artery disease (CAD) patients showed a 2.1\% improvement in FMD measured at $60 \mathrm{~min}$ after intake of non-alcoholized wine [20]. In another study involving CAD patients [21], FMD after consumption of $600 \mathrm{mg}$ of polyphenols from red wine peaked at $60 \mathrm{~min}$, which is similar to our results in elite athletes. However, maximal improvement in FMD (1.9\% increase from baseline) with polyphenol intake was slightly smaller than the change seen in our population of athletes $(2.8 \%$ increase from baseline). This difference in magnitude may be attributable to age differences in subjects between the two studies (mean age of 61 vs. 23 years), since endothelial function tends to deteriorate with aging [22]. Brachial artery FMD has been shown to be significantly greater in runners compared with sedentary controls and swimmers [23]. On the other hand, a recent study has shown that Olympic and world class athletes have lower superficial femoral artery FMD than controls, whereas in the brachial artery, a lower FMD was found in squash players [24]. Authors have hypothesized that this apparently lower artery FMD may be related to the profound structural remodeling in the diameter and wall thickness of the conduit arteries of athletes. It is not known if athletes that we have tested in our studies had enhanced FMD compared with sedentary subjects or to athletes from other sports. Thus, we cannot speculate on the extent to which the FMD response to polyphenol intake in our study is large or small considering their "baseline" status. Nevertheless, results from the present study suggest that even in highly trained athletes, consumption of $600 \mathrm{mg}$ of polyphenols from cranberries and grape seeds modifies FMD acutely.

Mechanisms underlying the acute FMD response to polyphenol intake have been proposed. There is in vitro evidence for changes in the phosphorylation pattern of endothelial nitric oxide synthase (eNOS) in response to tea polyphenols [25]. Consistent with these data, treatment of cultured bovine aortic endothelial cells (BAEC) with hesperetin, a citrus flavonoid, has been shown to acutely stimulate phosphorylation of eNOS to produce nitric oxide, a key signaling molecule involved in vasodilation. TNF$\alpha$ mediated adhesion of monocytes to BAEC has also been shown to be attenuated by treatment with hesperetin [26]. This suggests that pre-existing signaling pathways rather than changes in gene expression and protein synthesis are most likely responsible for the rapid and acute FMD response to a single dose of polyphenol [27]. 
Chronic intake of flavonols and antioxidants has been suggested to attenuate insulin resistance in various populations [28-30] but we are not aware of studies that have investigated this issue in the context of an acute consumption in highly trained individuals. Our results have shown that a single $600 \mathrm{mg}$ dose of a polyphenol mix from cranberries and grape seeds does not alter the glycemic and insulinemic response to carbohydrate intake in this population, even when FMD is significantly altered. It will be interesting to investigate if chronic intake of polyphenols has long-term effects on these parameters.

\subsection{Study 2- Polyphenols and Performance}

It was of interest as part of our second objective to investigate if modifying FMD is associated with enhanced performance. We found that acute intake of polyphenols in elite athletes had no impact on performance in a 3-km bike TT as well as on perceived exertion. Participants received strict recommendations regarding intake of polyphenol-rich foods and supplements during the study. Based on the randomized cross-over nature of the study and its overall short duration (one week separated the two test days), usual dietary intake of polyphenols from polyphenol-rich foods is unlikely to be a confounding factor in our study.

The absence of an effect on performance can be attributed to several factors. First, it is possible that the dose of polyphenols used $(800 \mathrm{mg}$ ) may have led to changes in vascular function that are too small in magnitude to influence performance on a maximal test. Second, it has been shown that vascular function deteriorates in soccer players during the playing season, and returns to normal levels after a four-week recovery period [31]. Study 2 was undertaken when most athletes had just started their training regimen after a one-month rest. Since vascular function may degrade as the training amplifies during the season, testing the impact of polyphenol intake on performance during a more intense training period is of great relevance. Finally, the duration of the TT at maximal intensity was approximately four minutes, which requires a significant anaerobic component. We cannot exclude the possibility that polyphenol supplementation through its vasodilating effect may affect performance in the context of longer duration events, in which the aerobic capacity becomes a more important performance-limiting factor. Accordingly, acute supplementation with polyphenols from Ecklonia cava in 23 college student volunteers significantly increased time to exhaustion on a bicycle compared with placebo [32]. This deserves further investigation in elite athletes.

On the other hand, our results also suggest that for a similar degree of effort, time on the ergocycle and perceived exertion, a single dose of polyphenols pre-exercise resulted in significantly lower blood lactate concentration and heart rate post effort. Results were relatively similar in men and women (not shown). The increase in time to exhaustion after intake of polyphenols from Ecklonia cava has been seen along with a non-significant trend towards a decrease in plasma lactate levels in college students [32]. In another study testing the effect of four-week supplementation with $800 \mathrm{mg}$ of a coffeeberry formulation (polyphenol content not provided), blood lactate concentrations after $10 \mathrm{~min}$ of recovery following a 60second vertical jump test were significantly reduced compared with placebo [33]. However, similar to our study, there was no significant effect of the supplementation on anaerobic performance [33]. In our study, 
blood lactate levels were also lower prior to the 3-km TT, i.e., just after the warm-up, when subjects had consumed the polyphenol-rich drink. Thus, we cannot exclude the possibilities that acute intake of polyphenols prior to maximal exertion, by facilitating blood flow, may also facilitate oxygen delivery, and stimulate aerobic pathways, thereby limiting the production of lactate through anaerobic pathways, or may facilitate lactate clearance, or both. This needs to be explored further in the future studies.

\section{Conclusion}

Data from the present study show that acute polyphenol supplementation improves endothelial function but has no impact on performance on a 3-km TT. The extent to which polyphenol supplementation affects recovery or stimulates oxygen delivery or both during maximal anaerobic as well as aerobic efforts deserve further investigations. Additional studies are also needed to see how chronic vs. acute intake of polyphenols in athletes affects performance using specific and recognized endpoints. Finally, polyphenols have well characterized antioxidant properties [1]. The impact of antioxidant supplementation on sports performance is highly debated, but it should be stressed that most studies on this topic have been conducted using relatively weaker antioxidant supplements such as vitamins [34-36]. It will be interesting to balance the FMD-related effects of polyphenols with their antioxidant-related effects on recovery and performance.

\section{Acknowledgments}

These studies were developed and supported by Speed Skating Canada through the Own the Podium Program towards the 2010 Olympic Games in Vancouver and by Atrium Innovation. We are grateful to the nurses and the laboratory staff of the Nutrition and Functional Foods Institute and of Laboratory of Science of Physical Activity for their technical assistance and the expert care provided to the participants. We also express our gratitude to the participants, without whom the study would not have been possible.

\section{Author's contribution}

$\mathrm{BL}$ and $\mathrm{CC}$ has designed and obtained funding for this study. PC was responsible for the screening and medical supervision of the study participants. AMB coordinated Study \#1and KL coordinated Study \#2. MEP was involved in manuscript preparation and revision. BL and KL performed statistical analyses, analyzed the data and wrote the manuscript, which was reviewed critically by all authors. PC are Research Scholars from the Fonds de la recherche en santé du Québec (FRSQ). Laval University, the employer of $\mathrm{BL}$ and $\mathrm{CC}$, has licensed the product tested in these studies to Atrium Innovations, for commercial purposes. It is currently commercialized as CranLoad ${ }^{\mathrm{TM}}$ Sports.

\section{Conflict of Interest}

Laval University, the employer of $\mathrm{BL}$ and $\mathrm{CC}$, has licensed the product tested in these studies $\left(\right.$ CranLoad $\left.^{\mathrm{TM}}\right)$ to Atrium Innovation, for commercial purposes. 


\section{References}

1. Yoshihara, D.; Fujiwara, N.; Suzuki, K. Antioxidants: benefits and risks for long-term health. Maturitas 2010, 67, 103-107.

2. Ghosh, D.; Scheepens, A. Vascular action of polyphenols. Mol. Nutr. Food Res. 2009, 53, 322-331.

3. Gonzalez, R.; Ballester, I.; Lopez-Posadas, R.; Suarez, M.D.; Zarzuelo, A.; Martinez-Augustin, O.; Sanchez de Medina, F. Effects of flavonoids and other polyphenols on inflammation. Crit. Rev. Food Sci. Nutr. 2011, 51, 331-362.

4. Jackson, M.J. Free radicals generated by contracting muscle: By-products of metabolism or key regulators of muscle function? Free Radic. Biol. Med. 2008, 44, 132-141.

5. Davison, G.; Gleeson, M.; Phillips, S. Antioxidant supplementation and immunoendocrine responses to prolonged exercise. Med. Sci. Sports Exerc. 2007, 39, 645-652.

6. Lawrence, M.E.; Kirby, D.F. Nutrition and sports supplements: Fact or fiction. J. Clin. Gastroenterol. 2002, 35, 299-306.

7. Atalay, M.; Lappalainen, J.; Sen, C.K. Dietary antioxidants for the athlete. Curr. Sports Med. Rep. 2006, 5, 182-186.

8. Powers, S.; Nelson, W.B.; Larson-Meyer, E. Antioxidant and Vitamin D supplements for athletes: sense or nonsense? J. Sports Sci. 2011, 29 Suppl 1, S47-S55.

9. Bassett, D.R., Jr.; Howley, E.T. Limiting factors for maximum oxygen uptake and determinants of endurance performance. Med. Sci. Sports Exerc. 2000, 32, 70-84.

10. Foster, C.; Rundell, K.W.; Snyder, A.C.; Stray-Gundersen, J.; Kemkers, G.; Thometz, N.; Broker, J.; Knapp, E. Evidence for restricted muscle blood flow during speed skating. Med. Sci. Sports Exerc. 1999, 31, 1433-1440.

11. Campbell, B.I.; La Bounty, P.M.; Roberts, M. The ergogenic potential of arginine. J. Int. Soc. Sports Nutr. 2004, 1, 35-38.

12. Goulet, J.; Nadeau, G.; Lapointe, A.; Lamarche, B.; Lemieux, S. Validity and reproducibility of an interviewer-administered food frequency questionnaire for healthy French-Canadian men and women. Nutr. J. 2004, 3, 13.

13. Moens, A.L.; Goovaerts, I.; Claeys, M.J.; Vrints, C.J. Flow-mediated vasodilation: A diagnostic instrument, or an experimental tool? Chest 2005, 127, 2254-2263.

14. Thijssen, D.H.; Black, M.A.; Pyke, K.E.; Padilla, J.; Atkinson, G.; Harris, R.A.; Parker, B.; Widlansky, M.E.; Tschakovsky, M.E.; Green, D.J. Assessment of flow-mediated dilation in humans: A methodological and physiological guideline. Am. J. Physiol. Heart Circ. Physiol. 2011, 300, H2H12.

15. Corretti, M.C.; Anderson, T.J.; Benjamin, E.J.; Celermajer, D.; Charbonneau, F.; Creager, M.A.; Deanfield, J.; Drexler, H.; Gerhard-Herman, M.; Herrington, D.; et al. Guidelines for the ultrasound assessment of endothelial-dependent flow-mediated vasodilation of the brachial artery: A report of the International Brachial Artery Reactivity Task Force. J. Am. College Cardiol. 2002, 39, 257-265. 
16. Hashimoto, M.; Kim, S.; Eto, M.; Iijima, K.; Ako, J.; Yoshizumi, M.; Akishita, M.; Kondo, K.; Itakura, H.; Hosoda, K.; et al. Effect of acute intake of red wine on flow-mediated vasodilatation of the brachial artery. Am. J. Cardiol. 2001, 88, 1457-1460.

17. Agewall, S.; Wright, S.; Doughty, R.N.; Whalley, G.A.; Duxbury, M.; Sharpe, N. Does a glass of red wine improve endothelial function? Eur. Heart J. 2000, 21, 74-78.

18. Whelan, A.P.; Sutherland, W.H.; McCormick, M.P.; Yeoman, D.J.; de Jong, S.A.; Williams, M.J. Effects of white and red wine on endothelial function in subjects with coronary artery disease. Internal Med. J. 2004, 34, 224-228.

19. Dohadwala, M.M.; Holbrook, M.; Hamburg, N.M.; Shenouda, S.M.; Chung, W.B.; Titas, M.; Kluge, M.A.; Wang, N.; Palmisano, J.; Milbury, P.E.; et al. Effects of cranberry juice consumption on vascular function in patients with coronary artery disease. Am. J. Clin. Nutr. 2011, 93, 934-940.

20. Karatzi, K.; Papamichael, C.; Aznaouridis, K.; Karatzis, E.; Lekakis, J.; Matsouka, C.; Boskou, G.; Chiou, A.; Sitara, M.; Feliou, G.; et al. Constituents of red wine other than alcohol improve endothelial function in patients with coronary artery disease. Coron. Artery Dis. 2004, 15, 485-490.

21. Lekakis, J.; Rallidis, L.S.; Andreadou, I.; Vamvakou, G.; Kazantzoglou, G.; Magiatis, P.; Skaltsounis, A.L.; Kremastinos, D.T. Polyphenolic compounds from red grapes acutely improve endothelial function in patients with coronary heart disease. Eur. J. Cardiovasc. Prevent. Rehabil. 2005, 12, 596-600.

22. Walther, C.; Gielen, S.; Hambrecht, R. The effect of exercise training on endothelial function in cardiovascular disease in humans. Exerc. Sport Sci. Rev. 2004, 32, 129-134.

23. Nualnim, N.; Barnes, J.N.; Tarumi, T.; Renzi, C.P.; Tanaka, H. Comparison of central artery elasticity in swimmers, runners, and the sedentary. Am. J. Cardiol. 2011, 107, 783-787.

24. Green, D.J.; Rowley, N.; Spence, A.; Carter, H.; Whyte, G.; George, K.; Naylor, L.H.; Cable, N.T.; Dawson, E.A.; DH, J.T. Why isn't flow-mediated dilation enhanced in athletes? Med. Sci. Sports Exerc. 2013, 45, 75-82.

25. Anter, E.; Thomas, S.R.; Schulz, E.; Shapira, O.M.; Vita, J.A.; Keaney, J.F., Jr. Activation of endothelial nitric-oxide synthase by the p38 MAPK in response to black tea polyphenols. J. Biol. Chem. 2004, 279, 46637-46643.

26. Rizza, S.; Muniyappa, R.; Iantorno, M.; Kim, J.A.; Chen, H.; Pullikotil, P.; Senese, N.; Tesauro, M.; Lauro, D.; Cardillo, C.; et al. Citrus polyphenol hesperidin stimulates production of nitric oxide in endothelial cells while improving endothelial function and reducing inflammatory markers in patients with metabolic syndrome. J. Clin. Endocrinol. Metabol. 2011, 96, E782-E792.

27. Sies, H. Polyphenols and health: Update and perspectives. Arch. Biochem. Biophys. 2010, 501, $2-5$.

28. Grassi, D.; Desideri, G.; Necozione, S.; Lippi, C.; Casale, R.; Properzi, G.; Blumberg, J.B.; Ferri, C. Blood pressure is reduced and insulin sensitivity increased in glucose-intolerant, hypertensive subjects after 15 days of consuming high-polyphenol dark chocolate. J. Nutr. 2008, 138, 1671-1676. 
29. Grassi, D.; Lippi, C.; Necozione, S.; Desideri, G.; Ferri, C. Short-term administration of dark chocolate is followed by a significant increase in insulin sensitivity and a decrease in blood pressure in healthy persons. Am. J. Clin. Nutr. 2005, 81, 611-614.

30. Grassi, D.; Necozione, S.; Lippi, C.; Croce, G.; Valeri, L.; Pasqualetti, P.; Desideri, G.; Blumberg, J.B.; Ferri, C. Cocoa reduces blood pressure and insulin resistance and improves endothelium-dependent vasodilation in hypertensives. Hypertension 2005, 46, 398-405.

31. Reinke, S.; Karhausen, T.; Doehner, W.; Taylor, W.; Hottenrott, K.; Duda, G.N.; Reinke, P.; Volk, H.D.; Anker, S.D. The influence of recovery and training phases on body composition, peripheral vascular function and immune system of professional soccer players. PLoS ONE 2009, 4, e4910.

32. Oh, J.K.; Shin, Y.O.; Yoon, J.H.; Kim, S.H.; Shin, H.C.; Hwang, H.J. Effect of supplementation with Ecklonia cava polyphenol on endurance performance of college students. Int. J. Sport Nutr. Exerc. Metabol. 2010, 20, 72-79.

33. Ostojic, S.M.; Stojanovic, M.D.; Djordjevic, B.; Jourkesh, M.; Vasiljevic, N. The effects of a 4-week coffeeberry supplementation on antioxidant status, endurance, and anaerobic performance in college athletes. Res. Sports Med. 2008, 16, 281-294.

34. Close, G.L.; Ashton, T.; Cable, T.; Doran, D.; Holloway, C.; McArdle, F.; MacLaren, D.P. Ascorbic acid supplementation does not attenuate post-exercise muscle soreness following muscle-damaging exercise but may delay the recovery process. Br. J. Nutr. 2006, 95, 976-981.

35. Thompson, D.; Williams, C.; Garcia-Roves, P.; McGregor, S.J.; McArdle, F.; Jackson, M.J. Post-exercise vitamin $\mathrm{C}$ supplementation and recovery from demanding exercise. Eur. J. Appl. Physiol. 2003, 89, 393-400.

36. Avery, N.G.; Kaiser, J.L.; Sharman, M.J.; Scheett, T.P.; Barnes, D.M.; Gomez, A.L.; Kraemer, W.J.; Volek, J.S. Effects of vitamin E supplementation on recovery from repeated bouts of resistance exercise. J. Strength Cond. Res. Natl. Strength Cond. Assoc. 2003, 17, 801-809.

(C) 2013 by the authors; licensee MDPI, Basel, Switzerland. This article is an open access article distributed under the terms and conditions of the Creative Commons Attribution license (http://creativecommons.org/licenses/by/3.0/). 